\title{
Cell-SELEX Based Identification of an RNA Aptamer for Escherichia coli and Its Use in Various Detection Formats
}

\author{
Pooja Dua ${ }^{1,4}$, Shuo Ren ${ }^{2,4}$, Sang Wook Lee ${ }^{2,4}$, Joon-Ki Kim¹, Hye-su Shin ${ }^{1}$, OK-Chan Jeong ${ }^{3}$, Soyoun Kim ${ }^{2, *}$, \\ and Dong-Ki Lee ${ }^{1, *}$
}

\begin{abstract}
Escherichia coli are important indicator organisms, used routinely for the monitoring of water and food safety. For quick, sensitive and real-time detection of $E$. coli we developed a 2'F modified RNA aptamer Ec3, by Cell-SELEX. The 31 nucleotide truncated Ec3 demonstrated improved binding and low nano-molar affinity to $E$. coli. The aptamer developed by us out-performs the commercial antibody and aptamer used for $E$. coli detection. Ec3(31) aptamer based $E$. coli detection was done using three different detection formats and the assay sensitivities were determined. Conventional Ec3(31)-biotin-streptavidin magnetic separation could detect $E$. coli with a limit of detection of $1.3 \times 10^{6} \mathrm{CFU} / \mathrm{ml}$. Although, optical analytic technique, biolayer interferometry, did not improve the sensitivity of detection for whole cells, a very significant improvement in the detection was seen with the $E$. coli cell lysate $\left(5 \times 10^{4}\right.$ CFU/ml). Finally we developed Electrochemical Impedance Spectroscopy (EIS) gap capacitance biosensor that has detection limits of $2 \times 10^{4} \mathrm{CFU} / \mathrm{mL}$ of $E$. coli cells, without any labeling and signal amplification techniques. We believe that our developed method can step towards more complex and real sample application.
\end{abstract}

\section{INTRODUCTION}

Food and water contamination by pathogenic bacteria is a serious threat to the human health. Therefore, fast, accurate and economic methods of detection are critical to the timely diagnosis of virulent strains in the infected sources (Koedrith et al.,

\footnotetext{
${ }^{1}$ Global Research Laboratory (GRL) for RNAi Medicine, Department of Chemistry, Sungkyunkwan University (SKKU), Suwon 16419, Korea, ${ }^{2}$ Department of Bioengineering, Dongguk University, Seoul 04620, Korea, ${ }^{3}$ Department of Biomedical Engineering and School of Mechanical Engineering, Inje University, Gimhae 50834, Korea, ${ }^{4}$ These authors contributed equally to this work.
}

*Correspondence: dklee@ @skku.edu (DKL); skim@ dongguk.edu (SK)

Received 7 June, 2016; revised 1 October, 2016; accepted 18 October, 2016; published online 18 November, 2016

Keywords: aptamer, biolayer Interferometry, biosensor, cell-SELEX, Escherichia coli, impedimetric sensor
2015). As many pathogens are not easily detected, indicator organisms are a fundamental monitoring tool to assess the microbiological status of water and food (Tortorello, 2003). Detection of safety indicators suggest the presence of conditions associated with increased risk of exposure to a pathogen. These, along with yeasts, molds and some other bacterial species, also include Escherichia coli (Robbens et al., 2010). Although, most of the strains of $E$. coli found in food and water are not virulent, it is one of the most important bacterial targets for the monitoring of water and food safety because the existence of E.coli indicates that microbial contamination is high (Leclerc et al., 2001).

Conventional methods of E.coli detection that rely on traditional culture based assays, immunoassays or polymerase chain reaction based-assays (Arthur et al., 2005), are either time consuming and laborious or require sample processing, hence do not fulfill the requirement of real-time, on-site detection (Lazcka et al., 2007). Monitoring environmental and foodborne pathogens require quick, real-time disposable methodologies that are capable of detecting multiple analyte samples, as economically as possible. In this respect, biosensors have demonstrated a great potential in recent years (Arora et al., 2011). In addition biosensors offer possibilities of portability, miniaturization and on-site detection which are prerequisite in routine testing and sample screening by regulatory authorities and industries. Various kinds of biosensors (Nistor et al., 2002), namely, immunosensors (Park et al., 2008), electrochemical sensors (Li et al., 2014; Pandey et al., 2014), SPR based biosensors (Huang et al., 2011) have already been tested for pathogenic microorganisms. DNA and RNA aptamers have been shown to be ideal candidates for use as ligand in biosensors (aptasensors) (Amaya-Gonzalez et al., 2013; Wang et al., 2014) possessing many advantages over state of the art affinity sensors (Paniel et al., 2013). To this end, many ssDNA and RNA aptamers have been developed against pathogentic bacterias (Park et al., 2014; Suh et al., 2014) including E.coli O157:H7 (Kim et al., 2013; Lee et al., 2012; Wu et al., 2012). Technically, aptasensors can be fabricated to respond to any target molecule for which an aptamer can be generated. Herein, we report identification of 2'F modified RNA aptamer Ec3 for $E$. coli using Cell-SELEX against E. coli DH5 $\alpha$. Aptamer Ec3 binds specifically to $E$. coli but do not bind to gram negative bacterias of closly related Enterobacter or Serratia family. Also, no binding 
was seen with gram positive bacteria Bacillus subtilis. We then demonstrated the $E$. coli detection capacity of the minimized Ec-3(31) aptamer in three detection formats; conventional ligand pull-down and culturing assay, optical analytical technique biolayer inferometry and impedimetric biosensing, and compared the sensitivity of aptamer based detection in these assays.

\section{MATERIALS AND METHODS}

Unless otherwise noted, all chemicals were purchased from Sigma Aldrich or invitrogen. SELEX library (N40 library) was obtained from IDT and transcribed using DuraScribe ${ }^{\circledR}$ T7 Transcription Kit (Epicentre Biotechnologies, USA) following the manufacturer's protocol. Truncated aptamer Ec3(31), biotinylated or thiol modified oligonucleotides, HSR-1, SQ2 Mutant were chemically synthesized by ST pharma. Inc. Oligonucleotide sequence details are provided in Supplementary table 1. Lipoteichoic Acid (LTA) aptamer (OCT biotech, USA) was used as a positive control for gram positive bacteria and Lipopolysaccharide (LPS) aptamer (OCT biotech, USA) and E. coli biotin-antibody (ab68451, abcam, USA) were used as positive controls for gram negative bacteria

Microorganisms and culture conditions

E. coli DH5 $\alpha$, E. coli K12, E. coli KCTC2571, Enterobacter mori, Enterobacter arerogens and Serratia marcescens and B. subtilis were obtained from Korean Collection for Type Culture (KCTC). Cells were cultured in Luria broth. Fresh exponentially growing cultures (OD600 of 0.4) were used. For all experiments, cells were washed two times with wash buffer $(50 \mathrm{mM}$ Tris $\mathrm{pH}$ 7.4, $5 \mathrm{mM} \mathrm{KCl}, 100 \mathrm{mM} \mathrm{NaCl}, 1 \mathrm{mM} \mathrm{MgCl}$ ), before use.

\section{Cell-SELEX}

The starting 2'-F pyrimidine modified RNA library was prepared by in vitro transcription of a N40 DNA library containing a $40 \mathrm{nt}$ random region and primer binding sites (5'-ATACCAGCTTATTCAATT-N40-AGATAGTAAGTGCAATCT-3'), using Durascribe T7 transcription kit. 2 nmoles of this RNA library comprising of $10^{15}$ molecules were used for Cell-SELEX. The 2'-F RNA library was denatured in binding buffer $(50 \mathrm{mM}$ Tris $\mathrm{pH} 7.4,5$ $\mathrm{mM} \mathrm{KCl}, 100 \mathrm{mM} \mathrm{NaCl}, 1 \mathrm{mM} \mathrm{MgCl}, 0.02 \%$ Tween $20,0.1$ $\mathrm{mg} / \mathrm{ml}$ yeast tRNA and $1 \mathrm{mg} / \mathrm{ml} \mathrm{BSA}$ ) for $5 \mathrm{~min}$ at $95^{\circ} \mathrm{C}$. The $10^{8} \mathrm{E}$. coli cells were washed in wash buffer and incubated with the denatured library for $10 \mathrm{~min}$ at RT. The unbound sequences were partitioned by two successive washes in binding buffer for $5 \mathrm{~min}$ each, followed by centrifugation. The bound RNAs were eluted by heating at $95^{\circ} \mathrm{C}$ for $5 \mathrm{~min}$ and separated by phenol:chloroform:isoamyl alcohol extraction and ethanol precipitation. The PCl extracted RNA pool was reverse-transcribed using ImProm-II Reverse Transcription System (Promega) and 5 pmoles of $\mathrm{N} 40$ reverse primer ( $5^{\prime}$-AGATTGCACTTACTAT CT-3') and PCR amplified using N40 reverse and forward primer containing $T 7$ promoter (underlined) (5'-GGTAATACGACTCACTATAGGGAGATACCAGCTTATTCAATT-3') pairs. The purified PCR product was transcribed in vitro, PAGE purified and used for the next round. After 10 rounds of positive selection, two counter selections were carried out with $10^{\circ} \mathrm{B}$. subtilis cells and the unbound sequences were used for positive selections until the 12th round. Enrichment in the binders was quantified by real time qPCR, as described before (Dua et al., 2015). The 12th round, sequence pool was cloned using a TA cloning vector kit (RBC Bioscience Corp., Taiwan). 35 clones were sequenced and analyzed for consensus using Multialign soft- ware (Corpet, 1988).

\section{Aptamer characterization}

The screening of potential aptamers and calculation of binding affinity was done using qRT-PCR. The four selected aptamer sequences were in vitro transcribed and 25 pmoles of aptamers were incubated with $10^{8} \mathrm{E}$. coli or $B$. subtilis cells for $15 \mathrm{~min}$ at $\mathrm{RT}$. The bound RNA were heat eluted, $\mathrm{PCl}$ purified and quantitated by RT-PCR, relative to their respective input concentration standards. Aptamer three dimensional structure were predicted by RNA draw V1.1b2 (Matzura and Wennborg, 1996). For aptamer size minimization, serial truncated versions of Ec3 DNA oligo were annealed with the T7 promoter and in vitro transcribed to obtain 2 '-F modified truncated aptamers. The binding of these minimized forms in comparison to full length Ec3 was measured by qRT-PCR. Binding affinity of the final truncated version of Ec3 aptamers was calculated using the same method, except aptamers were tested at a concentration of $1 \mathrm{nM}$ to $500 \mathrm{nM}$. Experiments were repeated three times and the data has been represented as mean \pm SD

\section{E. coli Aptamer-bead pull-down assay}

Truncated aptamer Ec3(31) with $3^{\prime}$ biotin, SQ2 mutant-biotin and N40 primer-biotin controls, were allowed to bind to $20 \mu \mathrm{l}$ of streptavidin coated magnetic beads (dynabead, Invitrogen, USA) for $15 \mathrm{~min}$ at RT. yeast tRNA $(0.1 \mathrm{mg} / \mathrm{ml})$ and BSA (1 $\mathrm{mg} / \mathrm{ml}$ ) were added and the incubation was further continued for $45 \mathrm{~min}$. Unbound aptamer were washed-off and this preblocked bead-aptamer complex were incubated with various cell density of $E$. coli $\left(10^{8}\right.$ to $\left.10^{5} \mathrm{CFU} / \mathrm{ml}\right)$, in $1 \mathrm{ml}$ of binding buffer, for $30 \mathrm{~min}$ at RT. The E. coli-aptamer-magnetic bead complex was separated from the unbound cells on a magnetic stand (Invitrogen). The complex was washed 3-4 times and finally spread on a LB plate. Plates were incubated overnight and colonies were counted. To calculate the colony forming units $/ \mathrm{ml}(\mathrm{CFU} / \mathrm{ml})$, innoculum from serial dilutions of the starting $E$. coli cell concentration was plated on the LB plate. A standard curve of the colony counts vs initial microscopically determined cell concentration was plotted. Starting cell counts were converted to $\mathrm{CFU} / \mathrm{ml}$ and the number of colonies formed by the aptamer-bead pull-down, were plotted (data not shown). Experiments were repeated three times and represented as mean \pm SD.

Aptamer binding analysis by bio-layer interferometry (BLI) ForteBio's Octet RED 384TM instrument and streptavidin (SA) sensors (Pall life sciences, USA) (Cat: 18-5019) were used to study interactions between aptamer candidates and bacteria cells or bacteria lysates, according to manufacturer's instructions. All the aptamer target interactions were performed in binding buffer. In the case of antibody and control aptamers (LTA and LPS), 1X kinetic buffer (Fortebio) and buffer recipe provided by OTC biotech were used in accordance with the manufacturer's protocol. All aptamer candidates were heated at $95^{\circ} \mathrm{C}$, chilled on ice for $5 \mathrm{~min}$, and suspended in binding buffer at RT prior to use. SA sensors were pre-wet by binding buffer for $30 \mathrm{~min}$ followed by loading with aptamer candidates at 25ㅇ for $1200 \mathrm{~s}$ at $1000 \mathrm{rpm}$. After washing out nonspecific binding aptamers, sensors were blocked with biocytin and dipped into bacteria cells and lysates with desired concentrations. Association and dissociation profiles were measured for $10 \mathrm{~min}$. To monitor non-specific binding for biosensor, a reference sensor (without aptamer loading) was performed with the same procedure as aptamer loaded sensors. Kinetic screening results were 
Table 1. Aptamer sequences identified

\begin{tabular}{|c|c|c|}
\hline Sequence family & Aptamer sequence & $\begin{array}{l}\% \text { of total } \\
\text { sequences }\end{array}$ \\
\hline Ec3 & $\begin{array}{c}\text { AUACCAGCUUAUUCAAUUGCACGAAUUUGCUGUGUUUUUGGGGGGGUCGGGGAGU } \\
\underline{\text { AUAAGAUAGUAAGUGCAAUCU }}\end{array}$ & 20 \\
\hline Ec2 & AUACCAGCUUAUUCAAUUCUUCUUUGAUCUGCUCGUAUCAUGGGACGGGAGGGUA & 7 \\
\hline Ec5 & $\begin{array}{l}\text { AUACCAGCUUAUUCAAUUCGAAUUUAAGUUUCUUUUCUGGUAUAGUGGUUGGGUG } \\
\text { GGUAAGAUAGUAAGUGCAAUCU }\end{array}$ & 6.7 \\
\hline
\end{tabular}

acquired by subtraction from the reference sensor using Octet data analysis software, version 7.0 (ForteBio). In the case of aptamer inhibition, SA sensors were pre-coated with biotinylated truncated aptamers and then associated with lysates and unlabeled aptamer mixtures (pre-incubation at RT for $20 \mathrm{~min}$ before BLI assay).

\section{Impedance chip fabrication}

Two Cr/Au Gap capacitance electrodes of thickness $5 \mathrm{~nm}$ and $15 \mathrm{~nm}$ respectively were deposited on Pyrex glass (7740) by thermal evaporation process. The $\mathrm{Cr} / \mathrm{Au}$ layer were patterned and etched as shown in Fig. 4A. Parylene was deposited at 2 $\mu \mathrm{m}$ thickness at each electrode and patterned to form flow channels. One electrode makes inlet and outlet holes for sample introduction and removal, and two parylene coated electrodes are finally bonded by thermo-compressing bonding $\left(150^{\circ} \mathrm{C}\right.$, at $24 \mathrm{MPa}$ for $\left.20 \mathrm{~min}\right)$.

\section{Aptamer immobilization and impedance measurement} Thiol modified and Cy3 labeled Ec3 aptamer were mixed with $10 \mathrm{mM} \mathrm{NaCl}$. Four concentrations of aptamer solution were tested for immobilization ( $40 \mathrm{pM}, 0.4 \mathrm{nM}, 4 \mathrm{nM}$ and $18 \mathrm{nM}$ ) and incubated for 1 hour. The intensities of fluorescent signal, representing the aptamer immobilization capacity, were monitored. The unbound sample was washed by injection of PBST $(0.1 \%$ tween 20 in PBS buffer). The chip was heated to $90^{\circ} \mathrm{C}$ and slowly cooled down to allow proper aptamer secondary structure formation. The chip was washed with DI water thoroughly to remove the unbound aptamer. $1 \mathrm{mM}$ BSA solution was introduced into the channel and incubated for $30 \mathrm{~min}$. Serial dilutions of bacteria solution $\left(10^{8}, 10^{6}\right.$, and $\left.10^{4} \mathrm{CFU} / \mathrm{ml}\right)$ was introduced onto the chip. After $1 \mathrm{~h}$ of incubation, the chip was washed thoroughly and the bound bacteria were stained with Sybr Gold and visualized under fluorescent microscopy (Nikon eclipse TE2000-U). For impedance analysis, EIS measurement were performed which swept frequency from $1 \mathrm{~Hz}$ to $1 \mathrm{MHz}$ under low applied voltage (less than $0.01 \mathrm{~V}$ ) to avoid electrochemical reaction between electrode and liquid solution. As a negative control, we also measured impedance of buffer solution as a function of frequency.

\section{RESULTS AND DISCUSSION}

\section{Cell-SELEX based aptamer selection}

12 rounds of selections were performed to obtain $E$. coli binding 2'F modified RNA aptamers. In the last 2 rounds along with positive selections, two counter selections were also performed with gram positive bacteria $B$. subtilis. The enrichment of binders during selection process was measured using quantitative
RT-PCR. Starting library, $5^{\text {th }}$ round, $10^{\text {th }}$ round and $12^{\text {th }}$ round pool were tested for binding to $E$. coli. The SELEX pool showed increase in the percentage of binders with increasing cycles as compared to the starting N40 library (Fig. 1A) $12^{\text {th }}$ round pool showed significant enrichment of binders for $E$. coli. No binding was observed with $B$. subtilis (Fig. $1 \mathrm{~A})$. The $12^{\text {th }}$ round SELEX pool binding to $E$. coli was also confirmed by fluorescence microscopy using a TAMRA labeled reverse primer annealed to the pool (Supplementary Fig. 1). The pool was cloned and sequenced. Sequences were aligned into families according to sequence homology. As shown in Table 1, four distinct sequences were identified and their binding to $E$. coli was (2-15) times higher than that of initial library (Fig. 1B). Aptamer Ec3 demonstrates maximum binding to $E$. coli and was therefore chosen for further analysis. The secondary structure prediction of Ec3 shows a single prominent stem-loop structure in the random region (Fig. 1C) indicating the position of the binding domain. To minimize the aptamer size, serial truncations were performed keeping the probable binding loop intact. Binding analysis of these truncated nanostructures of Ec3, performed by quantitative PCR shows Ec3(31) as a minimum sequence length required for $E$. coli binding (Supplementary Fig. 2). Saturation curves of Ec3 plotted at various concentrations demonstrate a Kd of $225 \mathrm{nM}$.

\section{E. coli apta-magnetic pull-dlown}

In order to check the potential of aptamer Ec3 as an E. coli detection tool, we used a conventional enrichment and culturing method. Streptavidin coated magnetic beads probed with $\mathrm{Ec}(31)$-biotin were used to pull-down $E$. coli from cell suspension ranging from a concentration of $10^{8}-10^{5} \mathrm{CFU} / \mathrm{ml}$. The aptamer-bead-E. coli complex obtained after magnetic separation was cultured on LB plate and the colonies obtained were counted. N40 down primer-biotin (20 nt) and SQ2-mutant-biotin (30 nt) were used as controls. As can be seen in Fig. 2, Ec3(31) can efficiently pull-down $E$. coli from the cell suspension, in comparison to the control sequences. However, the limit of detection was $1.3 \times 10^{6} \mathrm{CFU} / \mathrm{ml}$. The aptamer could not detect $E$. coli from samples below this concentration. In addition this assay takes a little less than $24 \mathrm{~h}$ to obtain the results. The poor sensitivity of the assay is mainly due to detection via culturing. Replacing culturing with polymerase chain reaction based detection, post magnetic separation, can significantly improve the sensitivity, as shown in earlier studies (Lee et al., 2009; Ozalp et al., 2014). However, this method is time consuming and is not suitable for real-time detection.

Biolayer interferometry for $E$. coli detection

Biolayer interferometry or BLI is an optical analytical technique 
Aptamer Based Biosensor for Escherichia coli Detection

Pooja Dua et al.

A

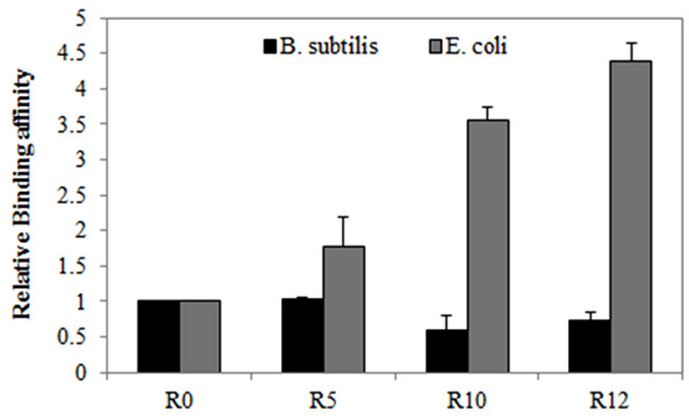

$\mathbf{B}$

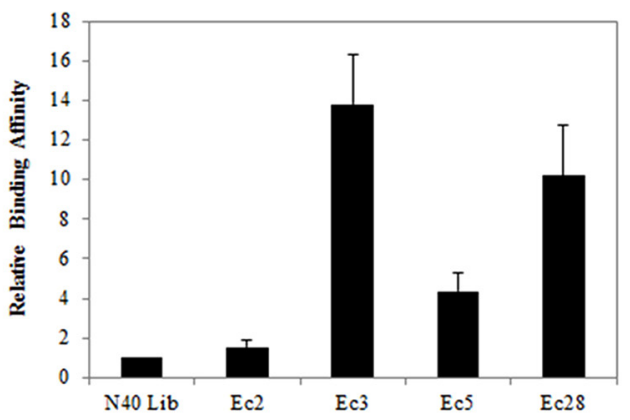

C

D
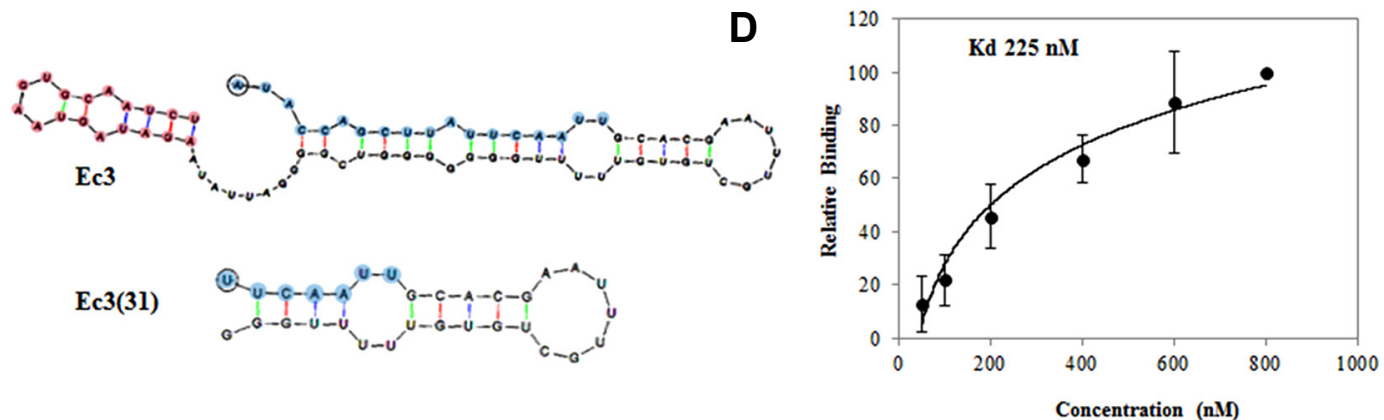

Fig. 1. Cell-SELEX for E. coli. (A) Progress of SELEX and enrichment of the binders. Quantitative PCR was used to monitor the enrichment in the SELEX pool. Binding relative to the N40 library is shown. (B) Binding of the four selected aptamers relative to N40 library. (C) RNA Draw predictions of the secondary structure of full length Ec3 and the truncated version Ec3(31). The upstream and downstream primer binding sequences are marked in blue and pink circles respectively. (D) The concentration dependent saturation curve for Ec3(31) shows binding affinity of $225 \mathrm{nM}$. Results are represented as mean \pm SD of 3 independent experiments.

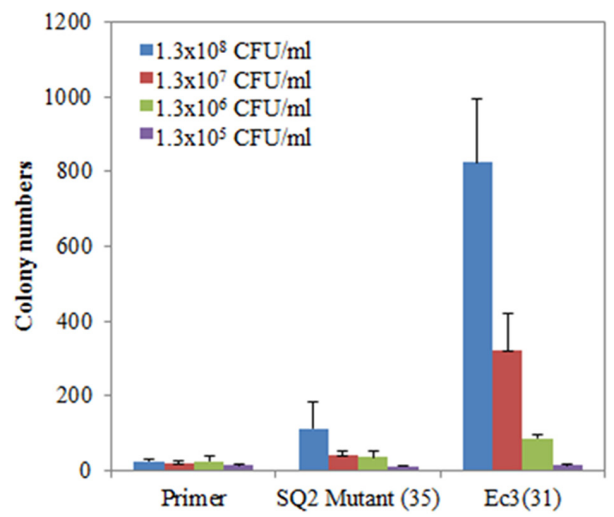

Fig. 2. Aptamer Ec3(31) mediated E. coli pull-down. Streptavidin coated magnetic beads complexed with Ec3(31)-biotin was used to pull-down $E$. coli from various concentrations of sample preparations and further cultured on LB plate. Colonies were counted and represented on the $Y$ axis. N40 down primer and SQ2 mutant sequences were used as controls. Results are represented as mean \pm SD of 3 independent experiments.

that allows real-time monitoring of molecular binding events occurring on the surface of the biosensor (Concepcion et al., 2009). BLI has been shown to be an efficient label-free assay format to detect molecular interactions. (Kammer et al., 2014;
Zichel et al., 2012). It analyzes the pattern of white light reflected from two surfaces: a layer of immobilized protein on the biosensor tip, and an internal reference layer. Any change in the number of molecules bound to the biosensor tip causes a shift in the interference pattern that can be measured in realtime. Use of BLI in detection of whole pathogens is a very novel approach and not many studies have been done in this regard. In a recent and probably the first study where BLI has been used to detect whole bacteria, gram-negative bacterium Francisella tularensis, the causative agent of tularemia was detected using a antibody based sanwich assay (Mechaly et al., 2016). We used BLI to check the binding of Ec3(31) to E. coli in comparison with some negative controls (blocked sensor, HSR-1, $\mathrm{N} 40 \mathrm{Lib}$ ) or positive controls that included an E. coli binding biotinylated antibody, and commercial aptamers against lipopolysaccharide and lipotechoic acid. The shift in the interference pattern was measured as association curves and the binding response was either plotted as a function of time or represented as a bar plot. As can be seen in Fig. 3A, aptamer Ec3(31) shows very strong binding to $E$. coli that clearly outperforms even the commercial $E$. coli detection tools. We then obtained the binding responses with various $E$. coli sample concentrations. As shown in Fig. 3B, with whole E. coli cells the limit of detection with BLI was similar to the magnetic separation-culturing method, i.e $1 \times 10^{6} \mathrm{CFU} / \mathrm{ml}$. We did not see any improvement in the sensitivity with the detection system in its current assay design. However, the current detection format is very basic and a much better sensitivity can be obtained using a sandwich assay where aptamer Ec31can act as a capture 
A

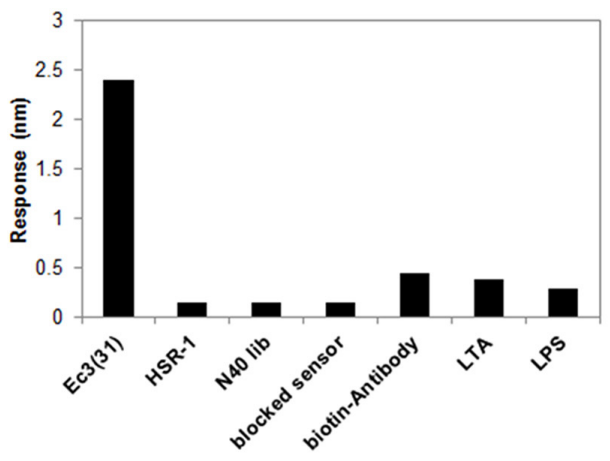

C

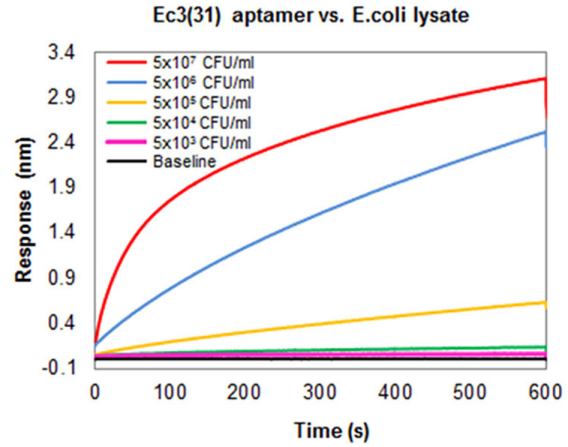

B
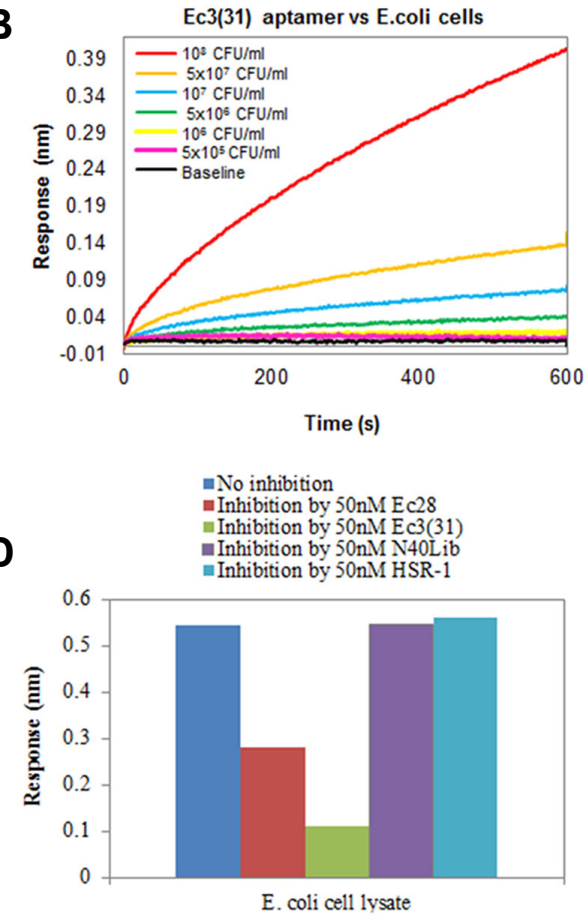

Fig. 3. Biolayer Interferometry based detection of E. coli. (A) The Octet sensor was probed with Ec3(31)-biotin or negative (N40, HSR-1, LTA aptamer) and positive controls ( $E$. coli antibody, LPS aptamer) and allowed to bind to $10^{7} \mathrm{CFU} / \mathrm{ml}$ of $E$. coli. The binding response is shown on the $Y$ axis. (B) Binding responses of Ec3(31) at various concentrations of $E$. coli cells (C) Ec3(31) binding responses for $E$. coli crude cell lysate prepared from varying cell numbers (D) Competition assay to confirm the specificity of the aptamer Ec3(31) binding to E. coli cell lysate. Results are representative of 3 independent experiments.

probe and a second aptamer or antibody may be used as a detection tool, as shown earlier (Mechaly et al., 2016). BLI is compatible with crude sample preparations; hence we did similar aptamer binding study with the crude E. coli cell lysate. As can be seen in Fig. 3C, sample processing could significantly improve the assay sensitivity to $5 \times 10^{4} \mathrm{CFU} / \mathrm{ml}$. In order to negate the possibility that increased binding response do not arise due to the non-specific interactions of the aptamer with crude $E$. coli lysate, some competition assays were performed. Briefly, free Ec3(31) aptamer, aptamer Ec28, N40 library or HSR-1 sequence were allowed to compete with the biotinylated Ec3(31) for the binding to E. coli lysate. Results in Fig. 3D shows that although free Ec3(31) could clearly compete with biotin-Ec3(31) for binding to $E$. coli, no competition was observed with $\mathrm{N} 40$ library or HSR-1 sequence. Some competition was also seen with aptamer Ec28, suggesting that Ec3 and Ec28 may bind to similar target on E. coli. This confirms that the specificity of Ec3(31) binding to $E$. coli is not affected by the sample processing and the increased binding responses may be solely due to better target presentation.

Aptamer immobilization on the impedimetric sensor electrode and $E$. coli binding efficiency test

To further improve upon the sensitivity of aptamer mediated $E$. coli detection, we took a label free electrochemical impedance spectroscopy approach. We fabricated a $\mathrm{Cr} / \mathrm{Au}$ Gap capacitance electrode based sensing device as shown in Fig. 4A. To find out optimal condition of aptamer binding on the electrode, we first investigated the binding ratio of the Ec3(31) aptamer on the electrode. Thiol-modified Сy3 labeled aptamer was first injected and incubated onto the chip. Four different concentration of the aptamer were injected $(40 \mathrm{pM}, 0.4 \mathrm{nM}, 4 \mathrm{nM}$ and 18 $\mathrm{nM}$ ) and incubated for $1 \mathrm{~h}$. Figure 4B shows aptamer binding efficiency on the Au electrodes. The intensities of fluorescent signal, representing the aptamer immobilization capacity, shows that over $4 \mathrm{nM}$ of aptamer concentration fluorescent signals were saturated (Fig. 4B) Therefore, aptamer concentration of $4 \mathrm{nM}$ was chosen as working concentration for impedance measurement. The Au electrode coated with the aptamer Ec3(31) was allowed to bind to E.coli cell ranging from $10^{8}$ to $10^{4} \mathrm{CFU} / \mathrm{ml}$ for $1 \mathrm{~h}$. The bound bacteria were visualized under fluorescent microscopy. A concentration dependent binding of E. coli was observed on the Ec3(31) coated Au electrode (Fig. 4C).

Impedimetric analysis of E. coli binding to the sensor Nyquist plot from impedance spectroscopy of various concentrations of bacteria solution and negative control are given in Fig. 4C. Each impedance signal is clearly different to that of negative control (No bacteria). Impedance increases with bacterial concentration. (Supplementary Fig. 4). In low frequency range such as $1 \mathrm{~Hz}$, impedance increase is caused by capacitance, since phase angle is close to - 90 deg. However, at higher frequency (over $1 \mathrm{kHz}$ ) resistance increases in proportion to the number of bacteria in solution. When frequency is over $10 \mathrm{kHz}$, impedance value is close to constant (Supplementary Fig. 4) and it increases in accordance with the density of bacteria concentration. It suggests that bacteria binding onto 
A

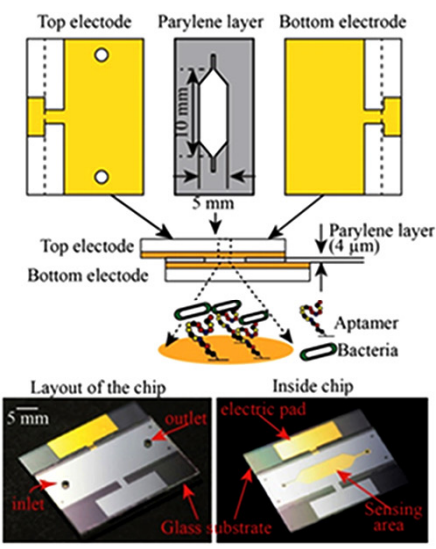

B

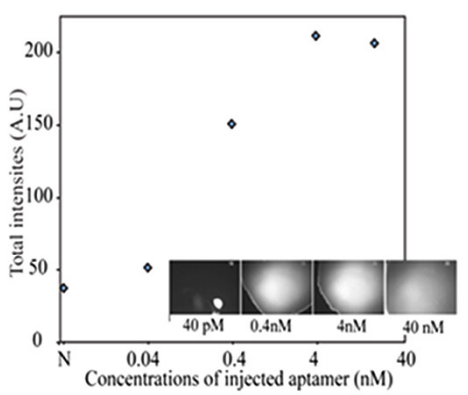

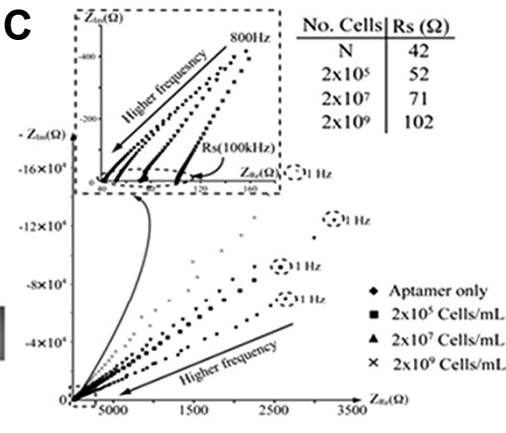

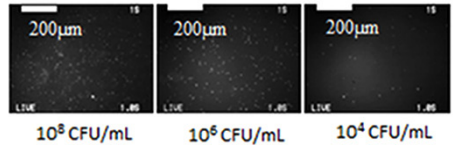

Fig. 4. Gap capacitance impedimetric sensor. (A) Layout of gap capacitance impedance sensor chip. Top and bottom electrode are bonded by parylene layer. The parylene layer also works as fluidic channel in-between the electrode. Layout of whole chip and real chip is shown below. (B) Aptamer immobilization. Thiol modified, cy3 tagged Ec3(31) was immobilized on the Au electrode. Various concentrations were used for coating the electrode. Immobilization efficiency was monitored by fluorescence measurement. (C) Nyquist plot from impedance analysis of $E$. coli. and microscopic visualization of bacteria on the chip. Bound bacteria was stained with Sybr Gold and visualized under fluorescent microscopy. Imaginary and actual impedance was plotted on the $Y$ and $X$ axis respectively. The high frequency area is being shown here. At higher frequency (over $1 \mathrm{kHz}$ ) resistance increases in proportion to the number of bacteria in solution.

the electrode work as resistance particles, inhibiting current flow between electrodes. The system that we developed here can be used as a sensor to monitor $E$. coli as it gives a bacterial concentration dependent response in a very rapid manner. Total experiment time is less than an hour from incubation of sample to measurement of signals. Especially, at higher frequency level (over $1 \mathrm{kHz}$ ), impedance signal is close to the constant value and it may indicate solution characteristics. The developed biosensor currently has detection limits of bacteria around $10^{4} \mathrm{CFU} / \mathrm{ml}$ without labeling and signal amplification techniques.

The 2'F modified RNA aptamer developed in this study has far better $E$. coli detection capacity than the standard commercial aptamers and antibody currently in use. The truncated and minimized aptamer sequence Ec3(31) can be easily chemically synthesized and functionalized as required. As the aptamer was developed using whole live $E$. coli cells, the nature and the identity of the aptamer target binding site is unknown. However, it seems that the aptamer binds to a component of the cell wall that is specific to Escherichia family, as no significant binding was seen with other related gram negative bacterias (Supplementary Fig. 3). We demonstrated the use of this aptamer as an $E$. coli diagnostic tool in various detection formats ranging from the conventional magnetic separation and culturing method, to optical and impedimetric sensing. To our knowledge, this is the first study where various aptamer based bacterial detection methods have been compared side by side. Among these methods, the maximum sensitivity of detection was observed with the gap capacitance based impedimetric sensor. The biosensor currently has detection limits of $2 \times 10^{4} \mathrm{CFU} / \mathrm{ml}$ of $E$. coli cells, without any labeling and signal amplification techniques and the overall time required for the detection is around an hour. Quick, sensitive detection of $E$. coli and easy portability of the sensor due to its miniaturized format altogether makes this aptasensor highly useful for on-site real time detection. We believe that our developed aptamer based sensing method can step-up towards more complex real samples (Brosel-Oliu et al.,
2015) .

Although our assay sensitivity is lesser in comparison to some of the impedance based detection of $E$. coli reported earlier, these studies have more advanced impedimetric set-up, that either uses electrode with better surface area (Akbari et al., 2015; Burrs et al., 2016), or have probes chemically conjugated to the electrode's surface (Guo et al., 2012). In other cases, the change in impedance is measured in terms of growth of bacteria on the electrode, rather than the antibody/antigen binding (Settu et al., 2015). Whereas, the main objective of our study was to test E. coli binding capacity of the aptamer and its compatibility to various detection systems, for which we preferred basic assay designs, with-out additional labels and amplification, to clearly understand the potential of this aptamer. Now when we have established its use, we can definitely move towards more advanced and sensitive biosensor platforms to attain the required sensitivity.

Note: Supplementary information is available on the Molecules and Cells website (www.molcells.org).

\section{ACKNOWLEDGMENTS}

This work was supported by Agency for Defense Development through Chemical and Biological defense Research Centre (no. UD140017ID). Pooja Dua was supported by Basic Science Research Program through the National Research Foundation of Korea (NRF) funded by the Ministry of Education (NRF 2013R1A1A2062908).

\section{REFERENCES}

Akbari, E.,Buntat, Z.,Afroozeh, A.,Zeinalinezhad, A., and Nikoukar A. (2015). Escherichia coli bacteria detection by using graphenebased biosensor. IET Nanobiotechnol. 9, 273-279.

Amaya-Gonzalez, S., de-los-Santos-Alvarez, N., Miranda-Ordieres, A.J., and Lobo-Castanon, M.J. (2013). Aptamer-based analysis: a promising alternative for food safety control. Sensors 13 , 16292-16311. 
Arora, P., Sindhu, A., Dilbaghi, N., and Chaudhury, A. (2011). Biosensors as innovative tools for the detection of food borne pathogens. Biosens. Bioelectron. 28, 1-12.

Arthur, T.M., Bosilevac, J.M., Nou, X., and Koohmaraie, M. (2005). Evaluation of culture- and PCR-based detection methods for Escherichia coli O157:H7 in inoculated ground beeft. J. Food Protection 68, 1566-1574.

Brosel-Oliu, S., Uria, N., Abramova, N., and Bratov, A. (2015). Impedimetric sensors for bacteria detection, biosensors - micro and nanoscale applications. In nanotechnology and nanomaterials. Biosensors - Micro and Nanoscale Applications", T. Rinken, ed.

Burrs, S.L., Bhargava, M., Sidhu, R., Kiernan-Lewis, J., Gomes, C., Claussen, J.C., and McLamore, E.S. (2016). A paper based graphene-nanocauliflower hybrid composite for point of care biosensing. Biosens. Bioelectron. 85, 479-487.

Concepcion, J., Witte, K., Wartchow, C., Choo, S., Yao, D., Persson, H., Wei, J., Li, P., Heidecker, B., Ma, W., et al. (2009). Label-free detection of biomolecular interactions using BioLayer interferometry for kinetic characterization. Comb. Chem. High Throughput Screen. 12, 791-800.

Corpet, F. (1988). Multiple sequence alignment with hierarchical clustering. Nucleic. Acids Res. 16, 10881-10890.

Dua, P., S, S., Kim, S., and Lee, D.K. (2015). ALPPL2 aptamermediated targeted delivery of 5-Fluoro-2'-Deoxyuridine to pancreatic cancer. Nucleic Acid Ther. 25, 180-187.

Guo, X., Kulkarni, A., Doepke, A., Halsall, H.B., lyer, S., and Heineman, W.R. (2012). Carbohydrate-based label-free detection of Escherichia coli ORN 178 using electrochemical impedance spectroscopy. Anal. Chem. 84, 241-246.

Huang, C.J., Dostalek, J., Sessitsch, A., and Knoll, W. (2011). Longrange surface plasmon-enhanced fluorescence spectroscopy biosensor for ultrasensitive detection of E. coli O157:H7. Anal. Chem. 83, 674-677.

Kammer, M.N., Olmsted, I.R., Kussrow, A.K., Morris, M.J., Jackson, G.W., and Bornhop, D.J. (2014). Characterizing aptamer small molecule interactions with backscattering interferometry. Analyst $139,5879-5884$

Kim, Y.S., Song, M.Y., Jurng, J., and Kim, B.C. (2013). Isolation and characterization of DNA aptamers against Escherichia coli using a bacterial cell-systematic evolution of ligands by exponential enrichment approach. Anal. Biochem. 436, 22-28.

Koedrith, P.,Thasiphu, T., Weon, J.I., Boonprasert, R., Tuitemwong K., and Tuitemwong, P. (2015). Recent trends in rapid environmental monitoring of pathogens and toxicants: potential of nanoparticle-based biosensor and applications. ScientificWorldJournal 2015, 510982.

Lazcka, O., Del Campo, F.J., and Munoz, F.X. (2007). Pathogen detection: a perspective of traditional methods and biosensors. Biosens. Bioelectron. 22, 1205-1217.

Leclerc, H., Mossel, D.A., Edberg, S.C., and Struijk, C.B. (2001). Advances in the bacteriology of the coliform group: their suitability as markers of microbial water safety. Ann. Rev. Microbiol. 55, 201-234.

Lee, H.J., Kim, B.C., Kim, K.W., Kim, Y.K., Kim, J., and Oh, M.K. (2009). A sensitive method to detect Escherichia coli based on immunomagnetic separation and real-time PCR amplification of aptamers. Biosens. Bioelectron. 24, 3550-3555.

Lee, Y.J., Han, S.R., Maeng, J.S., Cho, Y.J., and Lee, S.W. (2012).
In vitro selection of Escherichia coli O157:H7-specific RNA aptamer. Biochem. Biophys. Res. Commun. 417, 414-420.

Li, Y., Afrasiabi, R., Fathi, F., Wang, N., Xiang, C., Love, R., She, Z., and Kraatz, H.B. (2014). Impedance based detection of pathogenic E. coli O157:H7 using a ferrocene-antimicrobial peptide modified biosensor. Biosens. Bioelectron. 58, 193-199.

Matzura, O., and Wennborg, A. (1996). RNAdraw: an integrated program for RNA secondary structure calculation and analysis under 32-bit Microsoft Windows. Comput. Appl. Biosci. 12, 247249.

Mechaly, A., Cohen, H., Cohen, O., and Mazor, O. (2016). A biolayer interferometry-based assay for rapid and highly sensitive detection of biowarfare agents. Anal. Biochem. 506, 22-27.

Nistor, C., Osvik, A., Davidsson, R., Rose, A., Wollenberger, U., Pfeiffer, D., Emneus, J., and Fiksdal, L. (2002). Detection of Escherichia coli in water by culture-based amperometric and luminometric methods. Water Sci Technol. 45, 191-199.

Ozalp, V.C., Bayramoglu, G., Kavruk, M., Keskin, B.B., Oktem, H.A., and Arica, M.Y. (2014). Pathogen detection by core-shell type aptamer-magnetic preconcentration coupled to real-time PCR. Anal. Biochem. 447, 119-125.

Pandey, C.M., Tiwari, I., Sumana, G. (2014). Hierarchical cystine flower based electrochemical genosensor for detection of Escherichia coli O157:H7. RSC Adv. 4, 31047-31055.

Paniel, N., Baudart, J., Hayat, A., and Barthelmebs, L. (2013). Aptasensor and genosensor methods for detection of microbes in real world samples. Methods 64, 229-240.

Park, S., Kim, H., Paek, S.H., Hong, J.W., and Kim, Y.K. (2008). Enzyme-linked immuno-strip biosensor to detect Escherichia coli O157:H7. Ultramicroscopy 108, 1348-1351.

Park, H.C., Baig, I.A., Lee, S.C., Moon, J.Y., and Yoon, M.Y. (2014) Development of ssDNA aptamers for the sensitive detection of Salmonella typhimurium and Salmonella enteritidis. Appl. Biochem. Biotechnol 174, 793-802.

Robbens, J., Dardenne, F., Devriese, L., De Coen, W., and Blust, R. (2010). Escherichia coli as a bioreporter in ecotoxicology. App. Microbiol. Biotechnol. 88, 1007-1025.

Settu, K., Chen, C.J., Liu, J.T., Chen, C.L., and Tsai, J.Z. (2015). Impedimetric method for measuring ultra-low E. coli concentrations in human urine. Biosens. Bioelectron. 66, 244250.

Suh, S.H., Dwivedi, H.P., Choi, S.J., and Jaykus, L.A. (2014). Selection and characterization of DNA aptamers specific for Listeria species. Anal. Biochem. 459, 39-45.

Tortorello, M.L. (2003). Indicator organisms for safety and quality-uses and methods for detection: minireview. J. AOAC Int. 86 , 1208-1217.

Wang, H., Wang, Y., Jinghua, S.L., Xu, Y.W., Guo, Y., and Huang, J. (2014). An RNA aptamer-based electrochemical biosensor for sensitive detection of malachite green. RSC Adv. 4, 6098760994

Wu, W., Zhang, J., Zheng, M., Zhong, Y., Yang, J., Zhao, Y., Wu, W., Ye, W., Wen, J., Wang, Q., et al. (2012). An aptamer-based biosensor for colorimetric detection of Escherichia coli O157:H7. Plos One 7, e48999.

Zichel, R., Chearwae, W., Pandey, G.S., Golding, B., and Sauna, Z.E. (2012). Aptamers as a sensitive tool to detect subtle modifications in therapeutic proteins. PloS One 7, e31948. 